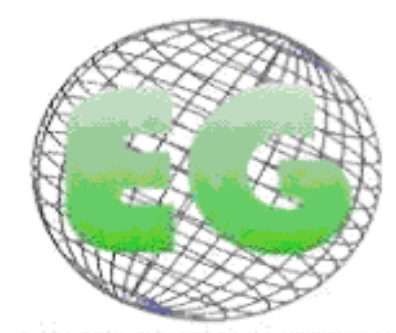

ISSN 1695-6141 $\mathbf{N}^{\circ} 25$
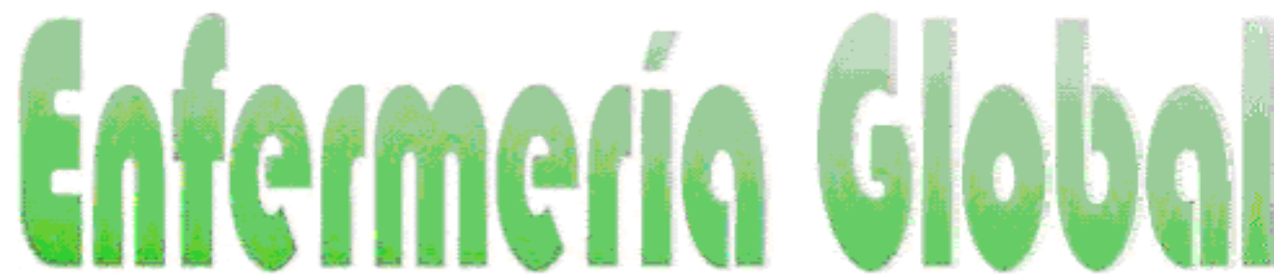

Revista electrónica trimestral de Enfermería

Enero 2012

www.um.eslegloball

REVISIONES

\title{
Volviendo a los planteamientos de la atención sensible
}

Retomando as abordagens do cuidado sensível

\author{
${ }^{*}$ Pereira, A., ${ }^{* *}$ Souza da Silva, R., ${ }^{* * *}$ De Camargo, CL., ${ }^{* * * *}$ Ribeiro de \\ Oliveira, RC.
}

\begin{abstract}
*Doutor em Enfermagem. Docente do PPGEnf da Escola de Enfermagem da Universidade Federal da Bahia. Líder do GECEN - Grupo de Estudos sobre o Cuidar em Enfermagem EEUFBA. E-mail: alvaro_pereira_ba@yahoo.com.br **Lcdo. em História. Mestrando em Enfermagem. Professor substituto da Escola de Enfermagem da Universidade Federal da Bahia. Secretário do GECEN. *** Doutora em Enfermagem. Docente do PPGENF da Escola de enfermagem da UFBA. Líder do CRESCER - Grupo de Estudos sobre o cuidado a criança e o Adolescente da EEUFBA. ${ }^{* * * *}$ Mestre em Enfermagem Médico-cirúrgica. Enfermeira do Hospital Universitário Professor Edgar Santos da UFBA. Docente de Enfermagem da Faculdade Dom Pedro II. Brasil.
\end{abstract}

Palabras clave: enfermería; cuidados de enfermería, humanización de la asistencia..

Palavras-chave: enfermagem; cuidados de enfermagem; humanização da assistência

Keywords: nursing; nursing care; humanization of assistance

\section{RESUMEN}

El cuidado sensible contempla la razón sensible sensible, la creatividad, la estética, la solidaridad, la libertad de asociación en relación con el cuidado humano.

Objetivos: Reflexionar sobre las demandas del cuidado de enfermería en el orden sensible y analizar los enfoques profesionales relativos a la sensibilidad en el cuidado.

Metodología: Se trata de un estudio reflexivo-analítico realizado a partir de una revisión bibliográfica sobre el cuidado sensible y la sensibilidad. Levantamiento realizado en la Biblioteca Virtual de Saúde - BVE, de publicaciones de los últimos diez años, se encontraron 23 publicaciones indirectas, en textos completos en la literatura nacional, identificados con los descriptores: Enfermería, Cuidados de Enfermería y Humanización de la Asistencia.

Resultados: El estudio reveló que el cuidado sensible a pesar de no ser descrito en la literatura de enfermería, se identifica con la solidariedad, amor y afectividad de los contactos profesionales; en la disponibilidad, en la entrega, en la escucha atenta; en el diálogo objetivo e interesado, en la 
conversación dedicada, em la conversación terapéutica o todo aquello que implica el diálogo afectivo en la práctica humanizada del cuidado.

Conclusiones: Los resultados destacan la necesidad de cambios de paradigma en el cuidado, de otra manera más orgánica, afectiva, más humana, que invierta en la subjetividad del ser, valorando lo vivido, la complejidad de los sentimientos que acompañan los desórdenes físicos y emocionales del usuario de los servicios de salud.

\section{RESUMO}

O Cuidado sensível contempla a razão sensível, a criatividade, estética, solidariedade, liberdade e parceria, na associação com o cuidado humanizado.

Objetivos: Refletir sobre demandas de cuidados de enfermagem na ordem sensível e Analisar as abordagens profissionais relativas à sensibilidade no cuidado.

Metodologia: Trata-se de um estudo reflexivo-analítico realizado a partir de uma pesquisa bibliográfica sobre o cuidado sensível e a sensibilidade. Levantamento realizado na Biblioteca Virtual de Saúde - BVE, de publicações dos últimos dez anos, foram encontradas 23 publicações indiretas, em textos completos na literatura nacional, identificados com os descritores: Enfermagem, Cuidados de Enfermagem e Humanização da Assistência.

Resultados: o estudo revelou que o cuidado sensível apesar de não ser descrito na literatura de enfermagem, identifica-se com a solidariedade, amor e afetividade dos contatos profissionais; na disponibilidade, na entrega, na escuta atentiva; no diálogo objetivo e interessado, na conversa dedicada, no papo terapêutico ou tudo aquilo que implica no diálogo afetivo na prática humanizada do cuidado.

Conclusões: Os resultados destacam necessárias mudanças de paradigma no cuidado, em direção a outra ordem mais orgânica, afetiva, mais humana, que invista na subjetividade do ser, valorizando o vivido, a complexidade dos sentimentos que acompanham as desordens físicas e emocionais do usuário dos serviços de saúde.

\section{ABSTRACT}

SensitiveThe sensible care includes sensitiveble reasoning, creativity, aesthetics, solidarity, freedom, partnership and,, by association, with the human care.

Objectives: To reflect on the problems of nursing in the sensitiveble care approach and analyze some ways forto approach professionals to approachon the sensitivity care.

Methodology: This is a reflective study conducted from a literature review ofin texts on about sensitiveble care and sensitivity. The survey usedwas done in the database in the Virtual of Health Library in the last decadeand found 23 full text publications in national literature., Descriptors related to: Nursing, Nursing Care, and Humanized Assistance.

Results: The study shows that sensitiveble care, although not a descriptor in the nursing literature, is expressed in solidarity, love and professional contacts, in the availability, dispositionsal, with one attentive listening, and interested in dialogue, in the conversation-dedicated therapy, and in everything that involves the affective dialogue.

Conclusions: The study revealedevoked the need for paradigm shifts in nursing care, towards a another order more organic, affective, more humane order, that invests in the subjectivity, that valuesvaluing the lived complexity of feelings that accompany the physical and emotional disorders of the user of the consumer's healthy service. 


\section{INTRODUCCIÓN}

El cuidar y el cuidado profesional constituyen la base de una actividad relacional. Aunque hayan sido vistos como eminentemente técnicos también deberían contemplar comportamientos de interés, compasión, afectividad, consideración, además de las acciones de alivio al sufrimiento humano, mantenimiento de la dignidad y el manejo adecuado en las situaciones de crisis y experiencias de vivir y morir. ${ }^{1}$

El cuidado profesional se ha identificado como intervenciones fundamentadas en los referenciales de vida y en la expresión de lo vivido de las personas involucradas en esta situación relacional entre el enfermero y la persona del usuario de los servicios de salud, aprovechando sus experiencias cotidianas. En las últimas décadas esta distinción en relación al cuidado profesional se va afirmando como referencia u objeto principal de nuestra acción profesional.

A pesar de las circunstancias que contribuyeron a construir la enfermería moderna no puede aceptarse que este cuidado sea banalizado, difamado o desvalorizado por constituir, a lo largo de los siglos, un modo de ser mayoritariamente femenino, ni tampoco aceptar sus orígenes confundidos con acciones simplistas, de bases teóricas poco fiables para el acto de cuidar 0 incluso ser relacionado con aquel cuidado que compone la esencia del ser humano y de dominio de cualquier persona. ${ }^{2}$

Acabamos un siglo donde el cuidado y los cuidados de enfermería emergen como la base epistemológica, dando un salto hacia la construcción de una disciplina sólida, tiempos después de comprender que, para cualquier profesión que centra su radio de acción en el ser humano, se hace necesario cambiar la dirección de su práctica, revisar su campo y los límites del trabajo en esta área, transformando la mentalidad de quien cuida, enseña, investiga y gerencia el cuidado, en su esencia, a partir de sumergirse en la dimensión subjetiva, en sus pensamientos y sentimientos.

El cuidar se presenta como una acción que está lejos de la razón técnica y científica, es eminentemente de relaciones, por encontrar sus bases en el proceso interactivo. Se traduce en la forma de atención, cortesía, delicadeza, prontitud en las solicitaciones y comunicación efectiva ${ }^{3}$. Parece distinguirse también por el respeto a las dimensiones socioculturales y espirituales de los sujetos, que interactúan entre sí y conviven con una diversidad de significados simbólicos, padrones, valores, modos de vida, sobre qué y cómo cuidar con calidad, apoyándose en las expresiones del cuidado humano, en la diversidad, en el universo de una colectividad, como preconizó Leininger ${ }^{4}$ en su marco sobre transculturización ${ }^{5}$.

En esa dirección, el enfoque que buscamos en la enfermería debería desviarnos de lo curativo para el cuidado modificando la idea de que el ser humano que cuidamos no debe ser asistido esencialmente sobre la óptica del modelo curativo. Este divide a la persona en segmentos y en esa fragmentación se desvaloriza su condición emocional y el contexto sociopolítico-económico-espiritual en que está insertado. Nadie precisa ser configurado como portador de una enfermedad, pero sí ser comprendido como persona que no tiene exclusivamente desequilibrios de 
orden psicobiológicos y es eso lo que precisamos reproducir en la formación de las(os) enfermeras(os).

En la práctica, el trabajo de la(o) enfermera(o) viene concentrándose en la identificación de los desequilibrios de orden psicobiológico, porque se profundizó en el conocimiento pactado en la evaluación y/o en el diagnóstico de las dimensiones de la enfermedad. Trabajando dentro de la tradición cartesiana, siempre estuvo preocupada con la compartimentación del cuerpo enfermo y el uso de instrumentos básicos que priman las sensaciones, lo que ayuda en el examen corporal, en la evaluación y en el diagnóstico objetivo y preciso de la enfermedad.

En enfermería se busca una evaluación que precisa estar fundamentada en el padrón de problemas o procesos vitales alterados, que vienen siendo identificados y tratados como una taxonomía internacional. Este código fue definido por la Nort American Nursing Diagnosis Association (NANDA). Así mismo a pesar de la complejidad de las demandas del ser humano ese es un padrón diagnóstico que se viene construyendo, para unificar y rotular un lenguaje mundial para la mayoría de los problemas de enfermería, de los usuarios de los servicios de salud, a partir de características definidoras y evidencias de identificación clínico-quirúrgicas, ya que se presenta como la tentativa de encontrar un lenguaje singular para ayudar también a enfermería a realizar diagnósticos y cuidar con calidad de personas con desequilibrios (desarmonías), transformando la patología y los diagnósticos médicos en problemas de colaboración al diagnostico de enfermería.

En tanto se observa que la(o) enfermera(o) no dispone de un referencial que la(o) ayude a aproximarse a las demandas subjetivas de salud, aquellas que tratan de la dimensión de los sentimientos de las personas. Hay una gran carencia de habilidades y conocimientos prácticos para cuidar de un conjunto de acciones definidas como sensible. Estas demandan cuidados relacionados a problemas de orden de sentimientos, fruto de la subjetividad, del mundo y de la vida, de la mayoría de las personas y que no tienen necesariamente relación directa con los cuidados corporales realizados.

De la vivencia de los autores en el área de cuidado de enfermería en los grupos GECEN (Grupo de Estudios sobre el Cuidar en Enfermería) y CRESCER (Grupo de Estudios sobre el cuidado al niño y adolescente), ambos pertenecientes a la Escuela de Enfermería de la Universidad Federal de Bahía, lo que fue observado es una preocupación en relación a los textos básicos usados en las disciplinas fundamentales de enfermería, con la formación de competencias y la ratificación de la dimensión instrumental en los cuidados corporales en detrimento de las dimensiones afectivas y espirituales.

En su operacionalización los instrumentos de evaluación tanto de los usuarios de los servicios de salud como de los gestores expresan la necesidad de la(o) enfermera(o) de dar mucha más atención a los datos objetivos, a los cuidados corporales y a la técnica por la técnica, de que a los datos subjetivos, aquellos con enfoque predominantemente afectivo, que utilizan los sentidos, las expresiones de los sentimientos, la emoción y aceptación en la relación personapersona; donde los cuidados realizados son caracterizados por una relación 
interpersonal efectiva, comprometida con el bienestar de la integralidad y el reequilibrio del otro.

Es evidente que vale más una enfermera con mucha habilidad y experiencia técnica, aun con el ceño fruncido y receptiva a los clientes, que otra que no siendo tan competente técnicamente, demuestre mayor preocupación con los sentimientos y demuestre afectividad y dedicación en los cuidados realizados. Ya se constata un cambio en la literatura nacional de enfermería sobre ese modelo de enfermería. El nuevo siglo ha traido una mayor preocupación por el ser humano, y aunque tarde, hemos visto mayor preocupación con las cuestiones ético-profesionales y personales, volviendo a la humanización de esos cuidados.

En ese contexto, este ensayo se justifica por la necesidad de evocar el estatus de profesión humanizada, haciendo necesario converger nuestros esfuerzos para la recuperación de los conocimientos sobre lo sensible y la sensibilidad. La razón sensible es un área del conocimiento que fue retirada por la cientificidad moderna, con la determinación de valorización de las dimensiones objetivas y de las sensaciones. En ese sentido, erigiendo de las cenizas como el Fénix, la razón sensible asume su merecido espacio profesional en la salud, a la vez que a lo largo de la modernidad fue totalmente desprestigiada, tornándose dependiente del saber y conocimiento de la clínica. Aquel saber dependiente que continúa separando el ser-cuidado en pedazos, como un gran mosaico de especialidades enfermas. Este conjunto se compone por una diversidad de conocimientos, que por su volumen de datos se torna casi inaccesible a la enfermería. Porque la omnisciencia del conocimiento de la clínica no viene siendo, una regla, una cualidad o un criterio usado en la excelencia del cuidado profesional de la enfermería.

En busca de esa excelencia precisamos invertir más en las formas comunicacionales, en las manifestaciones de lo dicho y no dicho, que se expresan en la afectividad de los contactos, en la disponibilidad y entrega; en el dialogo objetivo, coherente e interesado, en la conversación dedicada, terapéutica o en todo aquello que implica el dialogo efectivo; en la aproximación, observación rápida y cuidadosa, que requiere un "sexto sentido"6, en la percepción del conjunto ${ }^{7}$ y en la observación; en la escucha atenta; en el toque, en la dedicación, en la contemplación del ser, dentro de otros instrumentos, que aquí y allí vamos a precisar admitir como básicos para un cuidado sensible o como limitadamente hemos llamado de cuidado humano.

Todo cuidado 'dicho' humano se debería importar con la orden subjetiva de los cuidados - con los sentimientos, las demandas de sufrimientos del ser humano. Mas esa concepción viene decayendo en fase de una crisis ética, que privilegia el individualismo, que dejó de interesarse por el sufrimiento del otro, y acaba reflejandose también en los valores humanísticos que permean los cuidados en nuestra profesión ${ }^{8}$. Así, lo que se observa en la práctica profesional es que parece haber una crisis sobre la cuestión de la humanidad, que está intrínsecamente ligada al cuidado $\mathrm{y}$,

a pesar del cuidado humanizado ser conceptuado como respeto, amor, cariño, manteniendo el dialogo, la privacidad, dando atención a la familia, y representar una de las directrices de la asistencia de Enfermería, esto parece aún estar muy lejos de tornarse una realidad unánime. En 
general, el enfermero oye poco, habla mucho y no presta atención adecuada a los pacientes que se propone cuidar ${ }^{9} 579$.

En la práctica se constata que ese profesional incorporó la prepotencia y la omnisciencia, común a la mayoría de los profesionales del área biomédica, y viene subestimando la capacidad de comprender al ser humano en su plenitud como una persona que se construyó por sus experiencias de vida, su conocimiento, por sus hábitos, costumbres y sabidurías, que precisan ser respetados. Lo que parece una falta de reconocimiento ético personal y de la condición humana, además de la pérdida del carácter esencial de nuestra profesión.

Estamos vivenciando un modo de cuidado que está predestinado a ser reconocido como deshumanizado en su esencia y en ese sentido es posible deducir que la falta de humanización en las acciones del cuidado puede estar relacionada con la propia esencia de enfermería y la falta de valorización ética humana.

Así, destituido...

... de su sentido genuino, se quedó vacio de significado y se llenó de transparentes y/u oscuros conflictos, aquellos que acreditaban ser su carácter humano, la razón de la profesión. Luego se percibe que las atribuciones de enfermería dentro de un enfoque humanista no necesitan de cambios, sino de la inclusión de esta esencia ${ }^{10: 369}$.

Resultante de ese movimiento general en busca de la humanización en los espacios de atención, se observa que la racionalidad humana, aquella que privilegió lo científico en detrimento de lo humano, no está consiguiendo resistir las demandas de usuarios de los servicios de salud, que en la capacidad de movimiento de la calidad de los servicios viene imponiendo un nueva orden, más relacional, afectivo y atento con el ser cuidado.

La atención en ese sentido toma una forma sensible, y como un "cuerpo femenino" esas acciones asumen su primacía. Históricamente el cuidado nació en el espacio de la familia, siendo la mujer cuidadora consagrada por los hijos, ancianos, parientes y vecinos. Ella transmitió sus experiencias a las hijas y propagó por las comunidades el conocimiento del cuidar. Así las acciones del cuidado fueron incorporando la compasión, la comprensión, amor, acoger, tan común al comportamiento de las mujeres y, por otro lado, incompatibles con el modelo científico cartesiano, esencialmente masculino, adoptado en toda el área biomédica, porque este no favorece en su práctica los valores subjetivos ${ }^{11}$.

Aunque ya fue desprestigiada en el medio científico, por la ausencia de objetividad, es justamente por la primacía de la subjetividad humana que ella precisa ser reconocida; por su importancia en la resolución de los problemas que la razón científica no va dando cuenta de resolver. Ella es capaz de ser determinante en la formación de lazos afectivos, en las relaciones terapéuticas y en la perspectiva profesional, desde cuando rompe con la barrera de los compromisos personales y éticos mutuos, esenciales al movimiento de la cura biopsicosocial y espiritual y se asocia a la noción de que es en la estética y en lo 
sensible donde se puede hacer la redención profesional, en un mundo donde la asistencia a la enfermedad aun se muestra muy inhóspita ${ }^{12}$.

En esa dirección, nuestro objetivo con ese texto fue reflexionar sobre las demandas de cuidados de enfermería en el orden sensible y analizar los abordajes profesionales relativos a la sensibilidad, en la búsqueda de su entendimiento. Una modalidad próxima a la dimensión expresiva, que retrata un conjunto de formas del cuidar predominantemente cognitivo afectivo, prestado por los profesionales de enfermería al ser humano. De ese modo la sensibilidad es una noción que tiene origen en una razón mayor, la sensible. Ella es base para comprender los destinos de la condición humana, porque invierte en la subjetividad del ser y está en la historia, cotejando lo vivido, el sentimiento de los grupos humanos y es por eso que aquí merece ser destacada ${ }^{13}$.

En una aproximación inicial con la literatura, realizamos un levantamiento preliminar en las bases de datos científicos de enfermería, en busca de textos que traten de la sensibilidad y de la razón sensible en la enfermería. Como no fueron identificados descriptores directos sobre los dos temas, el levantamiento fue realizado con los descriptores enfermería, cuidados de enfermería, humanización de la asistencia, porque creemos que son los que más se aproximan a los temas propuestos en el análisis. En la base de datos Bireme - Biblioteca Virtual de Salud (BVS), en la última década, se evidenció 23 publicaciones on line de textos completos e indirectos sobre los temas en la literatura nacional.

Se identificó que la mayoría de estas publicaciones trata directa o indirectamente de los temas y llaman la atención por la necesidad de instituir inmediatamente acciones que contemplen la creatividad, estética, solidaridad, libertad y compañerismo por la inexorable e indispensable asociación con el cuidado y los fundamentos de la corriente humanística.

\section{EN BUSCA DE LOS FUNDAMENTOS TEÓRICOS DEL CUIDADO SENSIBLE}

La razón sensible parece haber dominado las tesis filosóficas de la antigüedad, con su mejor expresión en Platón, entre otros sabios, o en Pascal al final de la Edad Media. En la era moderna esta corriente de pensamiento se quedó arrinconada al dominio privado - en las artes, poesía, literatura, quedando despreciada por los científicos modernos, que buscaron en la objetividad, en las explicaciones y en las evidencias, los fundamentos para una conducción de los referenciales en las ciencias y en la salud. En general estos referenciales no han privilegiado los sentimientos, las emociones, la estética, el relato de los sufrimientos ajenos, el amor y la solidaridad, componentes esenciales de esa razón sensible ${ }^{13}$.

El agotamiento del paradigma moderno nos hace repensar los cambios en la formación y en la práctica profesional, de tal forma que los conocimientos hegemónicos, que efectuaron la penuria de esa sociedad, están con sus días contados, porque ellos no dieron cuenta de resolver la muerte, la desigualdad, el hambre y el desempleo, esclavizaron al hombre moderno alienándolo y condicionándolo a la producción y a la utilidad; otorgando mayor valor al orden, a la norma del trabajo; ${ }^{14}$ dando carácter subsidiario al descanso, a las vacaciones, a las fiestas, incluso habiendo sido ya reconocido el vitalismo e importancia del ocio en la creatividad por algunas empresas altamente productivas ${ }^{13,14}$. 
En cierta forma este paradigma desde hace algún tiempo viene agotándose y dando señales de que se está acabando, pues en vez de ayudar a la sociedad y hacer la vida del ser humano mas placentera, más feliz, manteniendo elevada la producción de endorfinas, reconocidamente benéficas para la salud, han contribuido a su enfermedad y hasta a la infelicidad.

Conviviendo paralelamente con este paradigma surge otro camino más subjetivo, de valor semejante, pero no menos significativo, el de la feminización y orientación de las costumbres y de los valores de los grupos humanos que establecen relación intrínseca con la concepción holística y fuerzan la ecolonización de la ciencia. Constituyese en una nueva forma de realizar ciencia, en una perspectiva ética que valora las cosas de significado menor, en una abordaje del cotidiano, que Maffesoli ${ }^{15}$ identificó como postmoderno.

Reconocer esa nueva ética al instante parece ser el desafío de quien convive con una orden inflexible para el proceso salud-enfermedad asociada a la tradición médica. Desde el inicio de la sociedad moderna, la medicina científica buscó sus fundamentos en la comprobación, en las evidencias, en las señales y en los exámenes, en aquello que puede ser visualizado por la inspección, medido por la palpación, auscultado con el auxilio de equipamientos, que están bajo el amparo de las normas, de los regímenes impuestos por los protocolos, de los consensos internacionales, programas institucionales, que construyen la objetividad, en detrimento de la subjetividad de las informaciones, de las experiencias anteriores, de la sensibilidad y de los sentimientos del ser.

La sensibilidad es ese orden subjetivo que se expresa por una sensación abstracta o intuitiva de aquello que conmueve, que se refina en la delicadeza de los sentimientos; de todo aquello que toca el corazón del ser humano y que los observadores sociales intentan categorizar o aprehender, mas no han sido capaces de precisar. Porque es imposible con los instrumentos modernos ser tocado. Ella es de la orden de la emoción, del afecto, sobrepasa la dimensión natural del sentimiento y la pasión. Ella toma fuerza en las construcciones y en la subjetividad colectiva que se expresa de forma creativa en las obras de arte, en los sentimientos colectivos, en las varias impresiones y valores que se manifiestan entre esas personas durante algunos periodos poco estudiados en la historia de la humanidad ${ }^{16}$.

Así, para expresar mejor su aprehensión, es preciso retomar los estudios que privilegian el dominio de la razón sensible y realizar análisis que den una necesaria profundización en la intuición, en el mundo simbólico, en los análisis de las representaciones imaginarias, en la ilusión, en la subjetividad de los sentimientos, en la pasión y empatía, en el amor y solidaridad, en la exploración fenomenológica del ser en su cotidiano. Es la emergencia de lo sensible y la sensibilidad que evocan en los sentidos intuitivos y todo lo demás que es de la orden del deseo, de los sentimientos y de la percepción pura del ser, en detrimento de los sentidos humanos que son co-sustanciales a las sensaciones y al deseo de controlar y aprender de los fenómenos, que son típicos de la orden cartesiana moderna ${ }^{13}$. 
Más que una razón ligada a los sentimientos, a la sensibilidad, es una "facultad de sentir compasión por la humanidad; piedad, empatía" siendo por tanto, una capacidad de "captar y expresar sentimientos y cosas" 17:681.

La subjetividad es esencialmente expresada por la producción del sentido, un sentido para las cosas de la vida y del cuidado. Ella no es solo una producción de sentido en el aspecto conceptual, sino una producción de efecto en la realidad de los sujetos deseosos. Las subjetividades, capitalista y religiosa, demuestran eso todo el tiempo, por intermedio de sus tendencias homogéneas de las singularidades ${ }^{18}$.

Lo sensible no es solo un momento insignificante en el cuadro referencial de un saber que viene depurándose, es el elemento central del conocimiento que permite estar en perfecta sintonía con la sensibilidad social, la cual siempre estuvo en cuestión, visto que esto es parte de la experiencia de lo vivido y de las relaciones empáticas entre grupos sociales.

La sensibilidad se refleja en las quejas, angustias y demás emociones, dudas, miedos, temores y tantos otros sentimientos presentes en la conversación de usuarios de los servicios de salud. Ella ha merecido ya hace algún tiempo preocupación especial de los docentes de enfermería, que desearían tener más condiciones de experimentar en la formación de esas(os) alumnas(os), experiencias subjetivas que conformen el carácter político, económico, éticoprofesional y humano del cuidado anhelado en la formación del enfermero, hasta no desperdiciar más nuestro tiempo discutiendo sobre el porqué el cuidado aún precisa estar siendo adjetivado de humano, cuando estamos esencialmente formados para prestar cuidados humanos y deberíamos estar obligados a atender los principios ético-profesionales para proporcionar un cuidado cualificado de esta naturaleza.

El cuidado sensible es una manera de cuidar semejante al cuidar expresivo, porque se fortalece en el aspecto cognitivo-afectivo, en el imperceptible. Un

“... momento de expresión de sentimiento, de la emoción del grado de aceptación o de rechazo. También, puede ser la demostración de carácter y de una conciencia internamente consistente. El eje conductor de cuidar es la esperanza en un momento en que los cuidados son realizados y caracterizados por una relación interpersonal" 19:749.

Es la primacía de los sentimientos en detrimento de las sensaciones. Esta última, al contrario de la sensibilidad, es reconocida por su dimensión objetiva y por las relaciones con el modelo médico.

Entendemos sensibilidad como una competencia subjetiva que precisa ser desenvuelta en la formación profesional. En principio, no todos los profesionales de salud reconocen las formas de intervenciones a los desequilibrios en la salud humana por la óptica de sensibilidad, mucho menos su valor científico. Eso no significa que en sus intervenciones esa sensibilidad no haya sido contemplada. Es un hecho que esa determinación está ayudando a usuarios de los servicios de salud a reconocer al mejor profesional del cuidado. Lo que puede ser fruto de una determinación para desarrollar habilidades especiales en el cuidado profesional y el deseo de reconocimiento de un usuario, satisfecho con la atención. 
En el mundo, el camino natural de la calidad en la prestación de servicios pasa por la realización de acciones ético-profesionales, comprensivas, comprometidas y la sensibilidad es la competencia profesional que imprime, precisamente, comportamiento y actitudes diferenciadas en esos profesionales. Esa competencia contempla el orden del sentimiento y de la subjetividad en sus acciones éticas, personales y de trabajo.

Es imposible dejar de reconocer la falta de empatía, la sordera emocional, la falta de una forma social en la interpretación de sentimientos ajenos; la sensatez, la sensibilidad o la indiferencia mecánica en la comunicación y actitudes; en la falta de sintonía, en los actos y cuidados prestados por profesionales, inhabilitados en el campo de las relaciones humanas, cuando existen entre ellos personas muy bien preparadas, y muy bien reconocidas técnico-científicamente.

Además de las quejas corporales el ser humano también expresa sufrimientos, angustias, temores, entre otros sentimientos que acompañan a los demás problemas físicos, psicológicos, sociales y espirituales en la mayoría de nuestros usuarios del sistema de salud. Así es imposible que sea la(el) enfermera(o) profesional, que podrá estar mejor preparado para identificar la subjetividad de esas expresiones y la resolución de esos problemas, que fueron vistos en su vivencia con el sufrimiento de los usuarios de los servicios de salud, por lo menos en las 24 horas de actividad diaria de su trabajo en el hospital.

En ese sentido, afirmamos que es la subjetividad una referencia profesional que asume su primacía, porque lo que estará en juego es la propia esencia de las cosas, los sentidos a ella atribuidos: en el sufrimiento, en la alegría y en el placer; en la victoria y decadencia; en un mundo simbólico que se construye y se destruye entre personas en su tiempo y espacio social ${ }^{20}$.

Eso significa una nueva y necesaria inversión científica en la formación de las(os) enfermeras(os) en las ciencias subjetivas dando énfasis a las dimensiones histórico-existenciales, estructurales-profesionales, psicoafectivas, ecológicoambientales, psicosocioculturales y espirituales que impregnan los cuidados a esos grupos humanos.

Asumen mérito semejante a los significados evidenciados sobre la enfermedad, en detrimento de la enfermedad y de las sensaciones que ayudan a diagnosticar la patología; la valorización de los sentimientos que se expresan delante del sufrimiento de la enfermedad, en detrimento de las señales y síntomas, así considerando lo multidimensional del ser y desmitificando la supervalorización de los cuidados corporales. Es extrapolar las visiones de mundo y sus multidimensiones físicas, metafísicas en relación al ser humano, reconociendo nuestra ignorancia en algunas de esas dimensiones, lo que ya ha exigido respuestas diferenciadas en nuestro cotidiano profesional. Lo que significa mayor énfasis a los conocimientos relativos a las ciencias humanas, de modo a obtener equilibrio con las demás áreas biomédicas.

Finalizando, hace que se retomen algunas cuestiones antiguas evocadas ${ }^{22}$ en otros momentos de reflexión profesional: será que en nuestra verdadera área de conocimiento es biomédica o humana? Si fuimos una mescla de esas áreas donde estamos fallando, en la construcción de ese cuidado sensible y más 
humano? Si quisiéramos ese enfoque más sensible en la formación, no vamos a necesitar encaminar mudanzas en los currículos y rever la equivalencia de esas áreas en los currículos y proyectos pedagógicos de enfermería?

\section{A MODO DE CONCLUSIONES}

Al reflexionar sobre el arte del cuidar con sensibilidad se evoca la necesidad de cambio en el paradigma que indique un redireccionamiento en el enfoque de los cuidados de enfermería, centrados en un orden mecánico, en dirección a otro orden más orgánico, porque este señala mejor el cuidado sensible, la subjetividad del ser en su proceso histórico cotejando lo vivido y los sentimientos de los grupos humanos ante los procesos de vida, enfermedad y muerte. Eso también puede requerir una nueva dirección en relación al desempeño de la(el) enfermera(o) sobre los modos de ser/hacer en enfermería, en busca de un cuidado cada vez mas relacional/afectivo, ético-solidario y de calidad, que contemple toda contribución ya erigida por las teorías humanísticas y ambientales de la enfermería, inspiradas en el legado de Nigthingale.

En la práctica somos profesionales preparados para rechazar el fracaso, cumplimos estándares, procedimientos, pero aún no tenemos una norma, o mejor, protocolos de enfermería, que indiquen cómo debemos actuar en la dimensión del cuidado sensible; en la atención de las demandas de cuidados de aquellos que enfrentan los sufrimientos provenientes de la enfermedad. $\mathrm{Ni}$ siquiera conseguimos cualificar algunos de los comportamientos esperados en el proceso del cuidado de aquellos usuarios de los servicios de salud, que buscan resolver los desequilibrios en sus procesos vitales, estando en condiciones reales o de riesgo en relación a los problemas de salud.

En ese sentido, encarando ese verdadero desafío es preciso comenzar a describir precisamente cuáles son los instrumentos "básicos", pero esenciales a los cuidados humanizados y cómo desarrollar nuestros antiguos sentidos en busca de una nueva sensibilidad, para vivenciar y relatar esas experiencias en la lucha con la complejidad de los sentimientos ajenos, las desarmonías y demás expresiones de la subjetividad que acompañan al desequilibrio de orden física y emocional en el ser humano, para poder perfeccionar nuestras prácticas en esa dirección.

Precisamos tener valentía para escribir sobre los sentimientos sin preocuparnos con la razón científica moderna, colocando a nuestros profesionales para que tengan experiencias y publiquen más entre sus pares sobre los cuidados relacionales, destacando la óptica subjetiva y sensible de esos cuidados. Aun cuando esto no tenga el debido reconocimiento de la comunidad científica.

En la intención de profundizar una reflexión sobre los problemas de enfermería, en este orden y búsqueda de entender algunas formas de abordajes profesionales, relativos a la sensibilidad, nos decantamos por el análisis sin pretensiones de una modalidad aparentemente joven, que está más cerca de la dimensión expresiva, porque ayuda a ese contexto predominantemente cognitivo afectivo, prestado por los profesionales de enfermería al ser humano. Es verdad que esta aun precisa ser bien estudiada y divulgada, por lo que esperamos que otros también se atrevan a profundizar y teorizar sobre la misma. 


\section{REFERENCIAS}

1. Waldow VR. Processo de enfermagem: teoria e prática. Revista gaúcha de Enfermagem. 1988; 9(1):14-22.

2. Boff I. Saber cuidar: ética do humano - compaixão pela terra. 5 ${ }^{\underline{a}}$ Ed. Petrópolis: Vozes; 2000.

3. Caetano JÁ, Soares E, Andrade LM, Ponte RM. Cuidado humanizado em terapia intensiva: um estudo reflexivo. Esc Anna Nery R Enferm 2007 jun; 11 (2): $325-30$

4. Leininger M.Teoria do cuidado transcultural. In Anais do seminário brasileiro de teorias de enfermagem; 1985 maio 20-24; Florianópolis(SC): UFSC. 1985. p.232252.

5. Gonzáles RMB, Beck CLC, Denardin Ml. Cenários de cuidados. Aplicação de teorias de enfermagem. Santa Maria-RS: Pallotti; 1999.

6. Cianciarrulo,IT.(Org.) Instrumentos básicos para o cuidar: um desafio para a qualidade de assistência. São Paulo: Atheneu;1996.

7. Matheus MC, Fugita RMI, SA AC. Observação em enfermagem In: Cianciarrulo,IT.(Org.) Instrumentos básicos para o cuidar: um desafio para a qualidade de assistência. São Paulo: Atheneu;1996.cap. 2

8. Arruda EPN, Marcelino SR. Cuidando e confortando. In: Nascimento-Schulze CM. Dimensões da dor no câncer. São Paulo: Edit. Robe; 1997. p.157-89.

9. Puggina, ACG Silva, MJP. A alteridade nas relações de enfermagem. Rev. bras. enferm. 2005, 58(5): 573-759.

10. Girondi MBR, Hames. O cuidar institucional da enfermagem na lógica da pós-modernidade. Acta Paul Enferm. 2007;20(3):368-72.

11. Santos I, Figueiredo N, Sobral V, Tavares C. Cuidado: construindo uma nova história de sensibilidade. Online Brazilian Journal of Nursing [Internet] 2002 [Citado $2009 \quad$ Nov 16]; 1(3):[8p.] Disponível em www.uff.br/nepae/obin103santosietal.htm

12. Urakasi MBM. Interconexão da sensibilidade e da razão do cuidar.[tese].São Paulo:Universidade Federal de Enfermagem. Escola Paulista de Medicina; 2000.

13. Pereira A. O quotidiano profissional do enfermeiro: das aparências às diferenças de gênero. [Tese] Florianópolis (SC) Programa de Pós Graduação em Enfermagem da UFSC; 1999.

14. Rezende A L M de. Quotidiano e saúde. In: Rezende A. L. M. de; Ramos F. R. S.; Patrício Z. M. (Orgs.) O Fio das Moiras: o afrontamento do destino no quotidiano da saúde. Florianópolis: EDUFSC; 1995.

15. Maffesoli M. A ética pós-moderna. Rev. Fac. Educ. Jan./Dez. 1991; 17(1/2):194-202.

16. Maffesoli M. Éloge de la raison sensible. Paris: Bernadr Grasset; 1996.

17. Houaiss A, Villar M S, Franco FMM. Minidicionário Houaiss da língua portuguesa. 3ㄹed. Rio de Janeiro: Objetiva; 2009. p.681.

18. Teixeira, Enéas Rangel Um ensaio teórico filosófico sobre o desejo no cuidado numa perspectiva interdisciplinar. Esc Anna Nery R. Enferm 2002 Ago; 6(2):305-17.

19. Coelho MJ. Maneiras de Cuidar em Enfermagem. Rev Bras Enferm 2006 nov-dez; 59(6): 745-51.

20. Martins CR. A Imaginação e sentidos no cuidado de enfermagem [tese].Florianópolis (SC): Universidade Federal de Santa Catarina. Programa de Pós-Graduação emEnfermagem; 1999. 
21. Santos I dos, Figueiredo N, Sobral V, Tavares C. Caring: building new history of sensibility. Online Brazilian Journal of Nursing. [serial on line ] 2002.[cited 2002] $1(3)$ : 1-8. Available from:URL www.uff.br/nepae/obin103santosietal.htm

22. Paim L, Trentini M. Enfermagem ciência ou profissão? Rev. Gaúcha de Enferm. 1992;13(1): 28-33. 